\title{
ON A CERTAIN RING OF FUNCTIONS OF TWO VARIABLES*
}

BX

J. F. RITT

The product of two functions of the type

$$
a_{1}(x) \alpha_{1}(y)+\cdots+a_{n}(x) \alpha_{n}(y)
$$

is a function of similar type. There arises thus the problem of determining all representations of any function (1) as a product of functions (1). $\dagger$ The present paper throws some light upon this problem, without coming near to a complete solution of it.

We shall limit ourselves to functions with analytic $a$ 's and $\alpha$ 's.

We write each function (1) in such a way that the number of terms, $n$, in it is a minimum. This is accomplished by getting rid of any linear dependence which may exist among the $a$ 's and among the $\alpha$ 's. When the expression (1) for a function has a minimum number of terms, we say that the function is in reduced form. The expression in reduced form is unique to within linear transformations upon the $a$ 's and $\alpha$ 's.

Our first result states that if $A=B C$, where

$$
A=\sum_{i=1}^{n} a_{i}(x) \alpha_{i}(y), B=\sum_{i=1}^{r} b_{i}(x) \beta_{i}(y), C=\sum_{i=1}^{\dot{ }} c_{i}(x) \gamma_{i}(y),
$$

with $A, B, C$ in reduced form, then every $b_{i} c_{i}$ is an algebraic combination of the $a$ 's; that is, every $b_{i} c_{j}$ satisfies an algebraic equation whose coefficients are polynomials in the $a$ 's with constant coefficients. Similarly, every $\beta_{i} \gamma_{i}$ is algebraic in the $\alpha$ 's.

It follows immediately that the ratio of any two $b$ 's, or of any two $c$ 's, is algebraic in the $a$ 's. If, for instance, $b_{1}=1$, a situation which can be brought about by multiplying $B$, and dividing $C$, by a function of $x$, then every $b$ and every $c$ will be algebraic in the $a$ 's. In short, in factoring a function (1) into similar functions, no new transcendencies are introduced.

It is a consequence of the above result that if a function has only one term, it cannot be the product of two functions, either of which, in reduced form, has more than one term.

- Presented to the Society, October 26, 1929; received by the editors November 8, 1929.

† I bave learned, in conversation with Professor Blichfeldt, that he also had thought of this question, and, in fact, quite a number of years ago. 
The algebraic dependence theorem reduces the general factorization problem to a problem in algebraic functions of several variables. It becomes a question of determining all representations of a function

$$
\sum_{i=1}^{n} a_{i}\left(u_{1}, \cdots, u_{p}\right) \alpha_{i}\left(v_{1}, \cdots, v_{q}\right)
$$

where each $a$ and each $\alpha$ is algebraic in all of its variables, as a product of functions of similar type.

In Section II, the existence of irreducible functions is proved. It is shown that

$$
1+x y+\phi(x) \psi(y),
$$

where $\phi$ and $\psi$ are transcendental, cannot be the product of two functions of type (1), each of which, in reduced form, contains more than one term.

\section{Algebraic Dependence}

1. Reduced form. It is plain that if the $a$ 's or $\alpha$ 's in (1) are not linearly independent, we can write the function (1) with fewer than $n$ terms. We shall prove that when the $a$ 's and $\alpha$ 's are linearly independent, then $n$ is a minimum.

The linear independence of the $a$ 's and $\alpha$ 's being understood, suppose that (1) has another expression,

$$
b_{1}(x) \beta_{1}(y)+\cdots+b_{m}(x) \beta_{m}(y) .
$$

We equate (1) and (2) and differentiate the resulting relation $n-1$ times with respect to $y$. Let $y_{0}$ be a point at which the wronskian of the $\alpha$ 's does not vanish. Putting $y=y_{0}$ in the $n$ relations secured above, we solve for the $a$ 's. We find each $a$ expressed as a linear combination of the $b$ 's with constant coefficients. Hence we cannot have $m<n$, else the $a$ 's would be linearly dependent. Thus $n$ is a minimum. We see also that, if we have two expressions of (1) in reduced form, the $a^{\prime}$ s and $\alpha$ 's in either expression are linear combinations of those in the other.

2. Algebraic dependence. We shall prove the following theorem:

THEOREM I. Let

$$
A=a_{1}(x) \alpha_{1}(y)+\cdots+a_{n}(x) \alpha_{n}(y),
$$

with all $a$ 's analytic in some circle $|x-\zeta|<\rho$ and all b's analytic in some circle $|y-\eta|<\sigma$. Let $A=B C$, where

$$
B=b_{1}(x) \beta_{1}(y)+\cdots+b_{r}(x) \beta_{r}(y), \quad C=c_{1}(x) \gamma_{1}(y)+\cdots+c_{s}(x) \gamma_{s}(y)
$$


with b's and c's all analytic in some area in $|x-\zeta|<\rho$ and $\beta$ 's and $\gamma$ 's all analytic in some area in $|y-\eta|<\sigma$. It is understood that $A, B, C$ are in reduced form. Then every $b_{i} c_{j}$ is algebraic in the a's, and every $\beta_{i} \gamma_{j}$ is algebraic in the $\alpha$ 's.

That is, for every $b_{i} c_{j}$, there exists, identically in $x$, a relation

$$
P_{m} b_{i}^{m} c_{j}^{m}+\cdots+P_{1} b_{i} c_{j}+P_{0}=0,
$$

where the $P$ 's are polynomials in the $a$ 's with constant coefficients, not all $P$ 's being identically zero. A similar relation holds for every $\beta_{i} \gamma_{j}$.

We shall prove that part of our statement which refers to the $a$ 's, $b$ 's and $c$ 's. The functions of $y$ are treated similarly.

The product of $B$ by $C$ is of the form

$$
\sum b_{i} c_{j} \beta_{i} \gamma_{j} \quad(i=1, \cdots, r ; j=1, \cdots, s) .
$$

Let

$$
\beta_{i_{1}} \gamma_{i_{1}}, \cdots, \beta_{i_{p}} \gamma_{i_{p}}
$$

be linearly independent, and such that every $\beta_{i} \gamma_{i}$ is a linear combination of them with constant coefficients. Then

$$
B C=S_{1} \beta_{i_{1}} \gamma_{i_{1}}+\cdots+S_{p} \beta_{i_{p}} \gamma_{i_{p}}
$$

where each $S$ is a linear combination of the functions $b_{i} c_{i}$, with constant coefficients.

The $S$ 's may be linearly dependent. Suppose, changing subscripts, if necessary, that

$$
S_{1}, \cdots, S_{q}, q \leqq p,
$$

are linearly independent, and that every other $S_{i}$ is a linear combination of them with constant coefficients. If $q<p$, let, for $i>q$,

$$
S_{i}=u_{i 1} S_{1}+\cdots+u_{i q} S_{q},
$$

with constant $u$ 's. We have

$$
B C=S_{1} \Sigma_{1}+\cdots+S_{q} \Sigma_{q},
$$

where each $\Sigma$ is a linear combination of the functions in (4). The $\Sigma$ 's are linearly independent, for, as each $\Sigma_{h}$ involves $\beta_{i_{h}} \gamma_{j_{h}}$, but no other $\beta_{i_{k}} \gamma_{j_{k}}$ with $k \leqq q$, a linear dependence among the $\Sigma$ 's would imply one among the functions of (4).

Thus $q=n$, and (7) is a representation of $A=B C$ in reduced form. We 
execute linear transformations upon the $a$ 's and $\alpha$ 's in $A$, in such a way as to have, for the new $a$ 's and $\alpha$ 's,

$$
\begin{array}{ll}
S_{i}=a_{i} & (i=1, \cdots, n), \\
\Sigma_{i}=\alpha_{i} & (i=1, \cdots, n) .
\end{array}
$$

Evidently, if the products $b_{i} c_{j}$ are algebraic in the new $a$ 's, they are also algebraic in the original $a$ 's.

We have, in (6) and (8), a system of linear equations which are satisfied by the $b_{i} c_{j}$ 's. Now every $b_{i} c_{j}$ can be expressed in terms of the $s+r-1$ products

$$
b_{1} c_{1}, b_{1} c_{2}, \cdots, b_{1} c_{s} ; b_{2} c_{s}, \cdots, b_{r} c_{s} \text {. }
$$

We have

$$
b_{i} c_{j}=\frac{\left(b_{1} c_{j}\right)\left(b_{i} c_{s}\right)}{b_{1} c_{s}} .
$$

If a $b_{i} c_{j}$ is not in (10), we replace it in (6) and (8) by its expression (11). The equations (6) and (8) go over into equations in the functions of (10). Multiplying those of the latter equations in which $b_{1} c_{8}$ appears in a denominator by $b_{1} c_{s}$, we find a system of $(p-q)+n=p$ algebraic equations

$$
E_{i}=0 \quad(i=1, \cdots, p),
$$

which are satisfied by the products (10). In the first $p-q=p-n$ of the equations (12) the coefficients are constants. In each of the last $n$ equations, an $a$ appears among the coefficients.

The remainder of our proof will be concerned with showing that (12) determines the products (10) as algebraic combinations of the $a$ 's.

Let us, for the moment, regard the $b_{i} c_{j}$ 's in (12) as any solutions of (12), rather than as the products of the fixed $b$ 's and $c$ 's in $B$ and $C$. That is, momentarily, $b_{i} c_{j}$ is not being regarded as a product. Consider any solution of (12) with $b_{1} c_{8} \neq \equiv 0$. Let a function $b_{1} \not \equiv 0$ be chosen arbitrarily. We can now determine $c_{1}, \cdots, c_{s}$ in such a way that $b_{1} c_{i}$ is the product of $b_{1}$ by $c_{i}$, $i=1, \cdots, s$, and, having $c_{s}$, we can determine $b_{2}, \cdots, b_{r}$ so that $b_{i} c_{s}$ is the product of $b_{i}$ by $c_{s}, i=2, \cdots, s$. Then the products $b_{i} c_{j}, i=1, \cdots, r$; $j=1, \cdots, s$, will satisfy (6) and (8). If, now, we consider the product

$$
\left(b_{1} \beta_{1}+\cdots+b_{r} \beta_{r}\right)\left(c_{1} \gamma_{1}+\cdots+c_{s} \gamma_{s}\right),
$$

where the $b$ 's and $c$ 's are those just determined and where the $\beta$ 's and $\gamma$ 's are the fixed functions appearing above, we see first that this product equals the second member of (5), with the original $b$ 's and $c$ 's replaced by the new ones, then that it equals the second member of (7), with the same substitu- 
tions, finally that it equals the fixed function $A$. Hence any solution of (12) with $b_{1} c_{0} \neq 0$ will give a representation of $A$ as a product of two functions of type (1).

We are going to discuss the totality of the solutions of (12), using the theory of systems of algebraic equations as presented by van der Waerden in his paper Zur Nullstellentheorie der Polynomideale.*

We consider the ideal, with coefficients in the field of the $a$ 's and all constants, of which the functions $E_{i}$ in (12) are the basis. We represent this ideal as the least common multiple of a finite number of primary ideals. There is one of these primary ideals whose polynomials all vanish for the fixed $b_{i} c_{j}$ 's in (3). We take this primary ideal, and form the prime ideal associated with it, that is, the set of polynomials in the field of the $a$ 's and constants, which are such that some power of every one of them is in the primary ideal.

Let $F_{1}, \cdots, F_{l}$ be a basis for the prime ideal. Naturally every solution of

$$
F_{1}=0, \cdots, F_{l}=0
$$

satisfies (12).

If (13) has only a finite number of solutions, then every $b_{i} c_{j}$ in any of its solutions is algebraic in the $a$ 's.

Thus our theorem will be proved if we can show that (13) has only a finite number of solutions. Suppose that (13) has an infinite number of solutions. Then certain of the quantities (10) can be chosen arbitrarily, and (13) determines the remaining quantities as algebraic combinations of these.

What we have to keep in mind, in a function-theoretic way, is this. If the arbitrary quantities (10) are represented by $z_{1}, \cdots, z_{\mu}$, there exist $s+r-1-\mu$ functions of $x, z_{1}, \cdots, z_{\mu}$, all analytic at a point $\left(\chi, \zeta_{1}, \cdots, \zeta_{\mu}\right)$ where $\chi$ is some point at which the $a$ 's are analytic, $\dagger$ such that, if the z's are taken as functions of $x$ analytic at $\chi$ and assuming, at $\chi$, values close to the $\zeta$ 's, we get a set of $s+r-1$ analytic functions of $x$ which satisfy (13) (also (12)). For each $x$, the $s+r-1-\mu$ functions above are algebraic in the $z$ 's.

If $b_{1} c_{8}$ does not correspond to one of the arbitrary $z$ 's, then the function of $x$ and the $z$ 's, found for $b_{1} c_{s}$, will not be identically zero, for (13) contains a special solution, that of the original quantities (10), in which $b_{1} c_{s}$ is not zero.

We shall keep $z_{2}, \cdots, z_{\mu}$ fixed, at constant values close to their $\zeta$ 's. We shall keep $z_{1}$ indeterminate, allowing it to range over the neighborhood

* Mathematische Annalen, vol. 96 (1927), p. 196.

$\dagger$ For $\zeta_{1}, \cdots, \zeta_{\mu}$ we can take any values not situated on certain singular manifolds. 
of $\zeta_{1}$. Then the quantities (10) go over into functions analytic in $x$ and algebraic in $z_{1}$. If $b_{1} c_{8}$ is among $z_{2}, \cdots, z_{\mu}$, we take care to give it a constant value distinct from zero. If $b_{1} c_{s}$ is not among $z_{2}, \cdots, z_{\mu}$, we choose $z_{2}, \cdots, z_{\mu}$ so that $b_{1} c_{s}$, as a function of $x$ and $z_{1}$, is not identically zero.

We have now a one-parameter family of representations of $A$ in the form $B C$. $^{*}$ For $b_{1}$, we take any function of $x$, analytic and not zero at $\chi$. This determines the remaining $b$ 's and $c$ 's as functions of $x$ and $z_{1}$. We write

$$
\begin{aligned}
& B=b_{1}(x) \beta_{1}+b_{2}\left(x, z_{1}\right) \beta_{2}+\cdots+b_{r}\left(x, z_{1}\right) \beta_{r}, \\
& C=c_{1}\left(x, z_{1}\right) \gamma_{1}+\cdots+c_{s}\left(x, z_{1}\right) \gamma_{s} .
\end{aligned}
$$

We shall prove that either two of the $b$ 's in (14), or else two of the $c$ 's, have a ratio which is not a function of $x$ alone. $\dagger$ Suppose that the contrary is true. Then, as $b_{1}$ is independent of $z_{1}$, every $b$ is independent of $z_{1}$. Then the $c$ 's cannot all be independent of $z_{1}$, for some $b_{i} c_{j}$ equals $z_{1}$. Hence $C$ is the product of $c_{1}\left(x, z_{1}\right)$ by a function of $x$ and $y$ alone. A similar fact is true of $A$. Then $A$, like $c_{1}\left(x, z_{1}\right)$, must vary with $z_{1}$. This contradiction proves our statement.

Hence there must be some value $x^{\prime}$ of $x$ which makes neither $B$ nor $C$ vanish identically in $y$ and $z_{1}$, and for which at least two of the $b$ 's, or two of the $c$ 's, become functions of $z_{1}$ which are linearly independent. We write

$$
A\left(x^{\prime}, y\right)=B\left(x^{\prime}, y, z_{1}\right) C\left(x^{\prime}, y, z_{1}\right),
$$

where, expressing $B$ and $C$ in reduced form as sums of products of functions of $y$ and functions of $z_{1}$, we have

$$
\begin{aligned}
& B\left(x^{\prime}, y, z_{1}\right)=\delta_{1}(y) d_{1}\left(z_{1}\right)+\cdots+\delta_{t}(y) d_{t}\left(z_{1}\right), \\
& C\left(x^{\prime}, y, z_{1}\right)=\epsilon_{1}(y) e_{1}\left(z_{1}\right)+\cdots+\epsilon_{u}(y) e_{u}\left(z_{1}\right) .
\end{aligned}
$$

$A\left(x^{\prime}, y\right)$ is not zero for every $y$. In (16), (17), we must have either $t>1$ or $u>1$, for since the $\beta$ 's are linearly independent, and also the $\gamma$ 's, the elimination of the linear dependences among the $b$ 's and $c$ 's of (14), with $x=x^{\prime}$, is sufficient for the conversion of $B$ and $C$ into reduced form.

We let

$$
\theta_{i}=\frac{\delta_{i}}{A\left(x^{\prime}, y\right)} \quad(i=1, \cdots, t)
$$

Then

$$
\left[\theta_{1} d_{1}\left(z_{1}\right)+\cdots+\theta_{t} d_{t}\left(z_{1}\right)\right]\left[\epsilon_{1} e_{1}\left(z_{1}\right)+\cdots+\epsilon_{u} e_{u}\left(z_{1}\right)\right]=1
$$

\footnotetext{
* $B$ and $C$ may not be in reduced form in these representations.

$\dagger$ What follows also proves that either $r>1$ or $s>1$.
} 
for every $y$ and $z_{1}$. We represent, from now on, the first and second factors of the first member of (18) by $P$ and $Q$ respectively.

Since the $d$ 's and $e$ 's are algebraic in $z_{1}$, there is a Riemann surface on which they are all uniform. Let $R$ be such a surface. Let $U$ be an area in the $y$-plane in which the $\theta$ 's and $\epsilon$ 's are all analytic. For every $y$ in $U, P$ and $Q$ are algebraic functions of $z_{1}$, uniform on $R$.

Suppose that some $d$ has a pole at a point on $R$, corresponding to the value $w$ of $z_{1}$. First, let $w$ be finite. Of all $d$ 's which have poles at this point, let $d_{i_{1}}, \cdots, d_{i_{m}}$ be those the order of whose poles is a maximum, and let their developments at the pole all begin with terms in $\left(z_{1}-w\right)^{h}$ with $h<0$. Then, for any $y$ in $U, P$ has a development at the point under consideration beginning with a term in $\left(z_{1}-w\right)^{h}$ of coefficient

$$
v_{i_{1}} \theta_{i_{1}}+\cdots+v_{i_{m}} \theta_{i_{m}},
$$

where the $v$ 's are constants. As the $\theta$ 's are linearly independent, there is an area $V$, in $U$, in which the coefficient does not vanish. For any $y$ in $V$, $P$ will have a pole at the point under consideration. A similar result holds for $w=\infty$.

Evidently, then, we can find an area $W$, in $U$, such that, for $y$ in $W$, $P$ has a pole where and only where one of the $d$ 's has a pole, and $Q$ has a pole where and only where one of the $e$ 's has a pole. We may furthermore suppose that the order of any of the poles of $P$ or of $Q$ is independent of $y$ (in $W$ ).

As either $t>1$ or $u>1$, we suppose, without loss of generality, that $t>1$. By (18), $P$ has a zero where and only where $Q$ has a pole. Hence the zeros of $P$, as $y$ ranges over $W$, are fixed points, and the order of any zero of $P$ is independent of $y$.

Consider any two values of $y$ in $W$. For each of these values, $P$ becomes an algebraic function of $z_{1}$. The two algebraic functions, having the same zeros and the same poles, have a constant ratio. That is, $P$ is a function of $y$ multiplied by a function of $z_{1}$. This contradiction of the fact that $P$, in reduced form, has $t>1$ terms, shows that (13) has only a finite number of solutions. Theorem I is proved.

3. Functions with one term. We prove the following theorem:

THEOREM II. Unity cannot be the product of two functions (1), either of which, in reduced form, contains more than one term.

Let $1=B C$, with $B$ and $C$ in reduced form. Arrange so that $b_{1}=1, \beta_{1}=1$. Then every $b$ and $c$ will be an algebraic combination of 1 , and hence will be 
a constant. Thus, if there were two $b$ 's, they would be linearly dependent. This proves our theorem.

4. Reduction of problem. Let $A=B C$, as in Theorem I. Suppose that $b_{1}=1, \beta_{1}=1$, so that the $b$ 's and $c$ 's are algebraic in the $a$ 's, the $\beta$ 's and $\gamma$ 's algebraic in the $\alpha$ 's. We suppose that $n>1$.

Let $a_{1}, \cdots, a_{p}$ be such that no algebraic relation exists among them, but that every other $a$ is an algebraic combination of them. Let $\alpha_{1}, \cdots, \alpha_{q}$ be a similar set of $\alpha$ 's. Then the $b$ 's and $c$ 's are all algebraic in $a_{1}, \cdots, a_{p}$, the $\beta$ 's and $\gamma$ 's algebraic in $\alpha_{1}, \cdots, \alpha_{q}$.

Let $w$ be any function of $x$ which is algebraic in $a_{1}, \cdots, a_{p}$. Let

$$
P_{0} w^{m}+\cdots+P_{m}=0 \text {, }
$$

where every $P$ is a polynomial in $a_{1}, \cdots, a_{p}$. We suppose (19) to be irreducible in the field of $a_{1}, \cdots, a_{p}$ and all constants.

We replace $a_{1}, \cdots, a_{p}$ in the $P$ 's by variables $u_{1}, \cdots, u_{p}$. Then $w$ becomes an algebraic function of the $u$ 's. The singularities of this algebraic function are characterized by algebraic relations among the $u$ 's. Hence, because $a_{1}, \cdots, a_{p}$ are algebraically independent, not all values of the $u$ 's which are values of $a_{1}, \cdots, a_{p}$ can be singularities of the algebraic function. Thus there is a branch of the algebraic function which is analytic for values of the $u$ 's which are values of the $a$ 's, and which reduces to the original function $w$ of $x$ when the $u$ 's are replaced by the $a$ 's.

Thus, to the relation $A=B C$, there corresponds a relation

$$
\begin{aligned}
& a_{1}\left(u_{1}, \cdots, u_{p}\right) \alpha_{1}\left(v_{1}, \cdots, v_{q}\right)+\cdots+a_{n}\left(u_{1}, \cdots, u_{p}\right) \alpha_{n}\left(v_{1}, \cdots, v_{q}\right) \\
& =\left[b_{1}\left(u_{1}, \cdots, u_{p}\right) \beta_{1}\left(v_{1}, \cdots, v_{q}\right)+\cdots+b_{r}\left(u_{1}, \cdots, u_{p}\right) \beta_{r}\left(v_{1}, \cdots, v_{q}\right)\right] \\
& \cdot\left[c_{1}\left(u_{1}, \cdots, u_{p}\right) \gamma_{1}\left(v_{1}, \cdots, v_{q}\right)+\cdots+c_{s}\left(u_{1}, \cdots, u_{p}\right) \gamma_{s}\left(v_{1}, \cdots, v_{q}\right)\right],
\end{aligned}
$$

all functions being algebraic in their arguments. The relation holds when each $u_{i}$ is replaced by $a_{i}(x)$, each $v_{i}$ by $b_{i}(x)$. The functions of the $u$ 's in (20) are all analytic for certain values of the $u$ 's which are values of $a_{1}(x), \cdots$, $a_{p}(x)$. A similar statement holds for the functions of the $v$ 's.

We say that (20) is an identity in the $u$ 's and $v$ 's.

If the $u$ 's are held fixed at values assumed by $a_{1}(x), \cdots, a_{p}(x)$ at any $x$, then (20) becomes an identity in the $v$ 's. If it did not, there would exist a non-identical algebraic relation among $\beta_{1}(y), \cdots, \beta_{q}(y)$. This means that (20) holds for arbitrary $v$ 's, if only the $u$ 's are replaced by the $a$ 's. Then, for any set at all of $v$ 's, (20) must be an identity in the $u$ 's. Thus (20) is an identity in the $u$ 's and $v$ 's.

Conversely, suppose that the first member of (20) has, identically, such 
a factorization as is displayed in the second member. As we saw above, no $b$ or $c$ can have a singularity for all values of the $u$ 's which are values of $a_{1}(x), \cdots, a_{p}(x)$. A similar fact holds for the $\beta$ 's and $\gamma$ 's in (20). Hence every factorization of the first member of (20) gives a factorization $A=B C$. Furthermore, if the $b$ 's, for instance, in (20), are linearly independent, the functions of $x$ obtained from them will be linearly independent.

Thus there is a perfect equivalence between the problem of finding all factorizations of the function $A$, and the problem of determining all representations of the algebraic function

$$
a_{1}\left(u_{1}, \cdots, u_{p}\right) \alpha_{1}\left(v_{1}, \cdots, v_{q}\right)+\cdots+a_{n}\left(u_{1}, \cdots, u_{p}\right) \alpha_{n}\left(v_{1}, \cdots, v_{q}\right)
$$

as a product of similar functions.

\section{IRREDUCIBILITY}

5. Statement of result. We shall prove the following theorem:

THEOREM III. A function

$$
1+x y+\phi(x) \psi(y)
$$

with $\phi$ and $\psi$ transcendental, cannot be the product of two functions of type (1), each of which, in reduced form, has more than one term.

In virtue of $\$ 4$, we have to prove that there exists no relation

$$
1+x y+u v=\left[\sum_{i=1}^{r} a_{i}(x, u) \alpha_{i}(y, v)\right]\left[\sum_{i=1}^{s} b_{i}(x, u) \beta_{i}(y, v)\right],
$$

with $r>1, s>1$, with every $a, \alpha, b, \beta$ algebraic in each of its variables and with each of the four sets of functions $a, \alpha, b, \beta$ linearly independent.

We assume a relation (21) to exist, and force a contradiction.

6. First step. We shall prove that there exists an expression for $1+x y+u v$

$$
\begin{aligned}
1+x y+u v= & {\left[\sum_{i=1}^{p} c_{i}(x) \gamma_{i}(y)+\sum_{i=p+1}^{r} c_{i}(x, u) \gamma_{i}(y, v)\right] } \\
& \cdot\left[\sum_{i=1}^{q} d_{i}(x) \delta_{i}(y)+\sum_{i=q+1}^{s} d_{i}(x, u) \delta_{i}(y, v)\right]
\end{aligned}
$$

where $c_{1}, \cdots, c_{p} ; d_{1}, \cdots, d_{q}$, independent of $u$, and $\gamma_{1}, \cdots, \gamma_{p} ; \delta_{1}, \cdots, \delta_{q r}$ independent of $v$, are such that

$$
1+x y=\left(c_{1} \gamma_{1}+\cdots+c_{p} \gamma_{p}\right)\left(d_{1} \delta_{1}+\cdots+d_{q} \delta_{q}\right) .
$$

The $c$ 's, $d$ 's, $\gamma$ 's and $\delta$ 's in (22) are linearly independent sets of algebraic functions. 
In (21), we arrange so that the $a$ 's and $b$ 's are supported by a single Riemann manifold in the space of $x$ and $u$, and, similarly, that all the $\alpha$ 's and $\beta$ 's have the same manifold. This amounts, for the $a$ 's and $b$ 's, for instance, to determining an algebraic function $P$ of $x$ and $u$ such that every $a$ and every $b$ is rational in $P, x$ and $u$.

It is possible, in an infinite number of ways, to take an area $M$ in the plane of $x$ such that, for $x$ in $M$ and for $u$ small, any branch of any $a$ or $b$ can be expanded in ascending integral or fractional powers of $u$, the coefficients being algebraic functions of $x$, analytic in $M$. In what follows we deal with such expansions of any properly associated set of branches of the $a$ 's and $b$ 's.

Let the lowest of all powers of $u$ which figure in the expansions of all the $a$ 's be $u^{\sigma}$. As the $\alpha$ 's are linearly independent, the first factor in the second member of (21) will have an expansion beginning with $u^{o}$. Let the lowest power in the expansions of the $b$ 's be $u^{h}$. Then, because $1+x y$ is present in the first member of (21), we must have $h+g=0$. We multiply the $a$ 's by $u^{-o}$ and the $b$ 's by $u^{o}$. We are thus able to, and do, assume that the developments of the $a$ 's and $b$ 's contain no negative powers of $u$, and that some $a$, as well as some $b$, begins with a term in $u^{0}$. The multiplications by $u^{0}$ and $u^{-\sigma}$ require us to place the $a^{\prime}$ 's and $b^{\prime}$ s on a manifold on which $u^{0}$ is uniform, as well as $P$, above.

Suppose that $a_{1}, \cdots, a_{p}$ begin with terms $A_{i}(x) u^{0}, i=1, \cdots, p$, that $b_{1}, \cdots, b_{q}$ begin with $B_{i}(x) u^{0}, i=1, \cdots, q$, and that the other $a$ 's and $b^{\prime} s$ begin with higher powers of $u$. It is understood that no $A_{i}$ or $B_{i}$ is zero for every $x$. Equating the terms in $u^{0}$ in both members of (21), we have

$$
1+x y=\left(A_{1} \alpha_{1}+\cdots+A_{p} \alpha_{p}\right)\left(B_{1} \beta_{1}+\cdots+B_{q} \beta_{q}\right) .
$$

It is permissible to assume that the $A$ 's, and likewise the $B$ 's, are linearly independent. For instance, if $A_{1}, \cdots, A_{j}(j<p)$, are linearly independent, and the other $A$ 's linearly dependent upon them, we subtract from $a_{j+1}, \cdots, a_{p}$ linear combinations of $a_{1}, \cdots, a_{j}$, with constant coefficients, in such a way as to get a new set $a_{j+1}, \cdots, a_{p}$, whose developments begin with positive powers of $u$. This, of course, would require a suitable change of $\alpha_{1}, \cdots, \alpha_{j}$. We assume, then, that the $A$ 's and $B$ 's are linearly independent.

We are going to prove that the ratio of any two $\alpha$ 's, if $p>1$, or of any two $\beta$ 's, if $q>1$, in (24), is independent of $v$. Assuming the contrary, let us suppose, as we may, that $p>1$ and that $\alpha_{2} / \alpha_{1}$ is not independent of $v$. Let $y_{0}$ be a value of $y$ for which $\alpha_{2} / \alpha_{1}$ becomes a non-constant function of $v$. We put $y=y_{0}$ in (24) and have 


$$
\begin{aligned}
1+x y_{0}=\left[A_{1} \alpha_{1}\left(y_{0}, v\right)+\cdots+\right. & \left.A_{p} \alpha_{p}\left(y_{0}, v\right)\right] \\
\cdot & {\left[B_{1} \beta_{1}\left(y_{0}, v\right)+\cdots+B_{q} \beta_{q}\left(y_{0}, v\right)\right] . }
\end{aligned}
$$

Now (25) gives a representation of $1+x y_{0}$ as a product of functions of $x$ and $v$ of type (1), one of the functions of $x$ and $v$ consisting of at least two terms. This contradicts Theorem II. Thus any two $\alpha$ 's, or any two $\beta$ 's, in (24), have a ratio independent of $v$.

We see also, from (24), that $1+x y$ equals $\alpha_{1} \beta_{1}$ multiplied by a function independent of $v$, so that $\alpha_{1} \beta_{1}$ is independent of $v$. In (21), we divide the first factor, and multiply the second factor, by $\alpha_{1}$. We have then*

$$
\begin{aligned}
1+x y+u v= & {\left[\sum_{i=1}^{p} a_{i} \gamma_{i}(y)+\sum_{i=p+1}^{r} a_{i} \gamma_{i}(y, v)\right] } \\
& {\left[\sum_{i=1}^{q} b_{i} \delta_{i}(y)+\sum_{i=q+1}^{s} b_{i} \delta_{i}(y, v)\right] }
\end{aligned}
$$

with algebraic, and linearly independent, $\gamma$ 's and $\delta$ 's.

We take now an area $N$ in the plane of $y$ such that all of the branches of the $\gamma$ 's and $\delta$ 's have, for $v$ small, expansions in ascending powers of $v$, with coefficients algebraic in $y$ and analytic throughout $N$. In what follows, we work with any properly associated set of branches of the $\gamma$ 's and $\delta$ 's.

If $v^{\sigma}$ is the lowest power of $v$ in the developments of the $\gamma^{\prime}$ s, then $v^{-\sigma}$ must be the lowest power in the $\delta$ 's, since the first member of (26) contains $1+x y$. But $g \leqq 0$, since $\gamma_{1}(y)=\gamma_{1}(y) v^{0}$. Similarly $-g \leqq 0$, so that $g=0$. That is, no $\gamma_{i}$ with $i>p$, or $\delta_{i}$ with $i>q$, starts with a negative power of $v$.

We shall show that it is permissible to assume that every $\gamma_{i}$ with $i>p$ and every $\delta_{i}$ with $i>q$ starts with a positive power of $v$. Suppose that $\gamma_{p+1}, \cdots, \gamma_{p+j}$ start with terms $C_{i}(y) v^{0}, i=p+1, \cdots, p+j$, that $\delta_{q+1}, \cdots, \delta_{q+k}$ start with terms $D_{i}(y) v^{0}$, while the $\gamma^{\prime}$ 's and $\delta^{\prime}$ 's with higher indices, if there are any, start with positive powers.

We shall prove that every $C_{i}$ is linearly dependent on $\gamma_{1}, \cdots, \gamma_{p}$, and every $D_{i}$ is linearly dependent on $\delta_{1}, \cdots, \delta_{q}$.

Suppose that

$$
\gamma_{1}, \cdots, \gamma_{p}, C_{p+1}, \cdots, C_{p+l}
$$

are linearly independent, but that all other $C$ 's, if there are any, are linearly dependent on the functions of (27). Similarly, suppose that

$$
\delta_{1}, \cdots, \delta_{q}, D_{q+1}, \cdots, D_{q+m}
$$

are linearly independent, but that all other $D$ 's, if there are any, are linearly

${ }^{*} \gamma_{1}(y)=1$. 
dependent on the functions of (28). Then, by subtracting suitable linear combinations of $\gamma_{1}, \cdots, \gamma_{p+l}$ from $\gamma_{p+l+1}, \cdots, \gamma_{p+j}$ (if the latter exist), we get a new set $\gamma_{p+l+1}, \cdots, \gamma_{p+i}$, which start with positive powers of $v$. This requires a modification of the first $p+l$ of the $a$ 's, but it is to be observed that the first $p$ of the $a$ 's, even if they should be changed, will start with zero powers of $u$, while $a_{p+1}, \cdots, a_{p+l}$ will start with positive powers. Similar remarks apply to the $\delta$ 's and the $b$ 's.

With these adjustments, we put $v=0$ in (26), and find

$$
\begin{aligned}
1+x y=\left[a_{1} \gamma_{1}+\cdots+a_{p} \gamma_{p}+a_{p+1} C_{p+1}+\cdots+a_{p+l} C_{p+l}\right] \\
\cdot\left[b_{1} \delta_{1}+\cdots+b_{q} \delta_{q}+b_{q+1} D_{q+1}+\cdots+b_{q+m} D_{q+m}\right] .
\end{aligned}
$$

Referring to the discussion of (24), we see that, because the sets (27) and (28) are linearly independent, the ratio of any two $a$ 's, or any two $b$ 's, in (29), is independent of $u$. But this cannot be, for $a_{p+1}$ and $b_{q+1}$ start with higher powers of $u$ than do $a_{1}$ and $b_{1}$.

Thus, the $C$ 's and $D$ 's are, respectively, linear combinations of the $\gamma$ 's and $\delta$ 's. By subtracting suitable linear combinations of $\gamma_{1}, \cdots, \gamma_{\nu}$ from $\gamma_{p+1}, \cdots, \gamma_{p+j}$ and linear combinations of $\delta_{1}, \cdots, \delta_{q}$ from $\delta_{q+1}, \cdots, \delta_{q+k}$, we may arrange so that all $\gamma_{i}$ with $i>p$ and all $\delta_{i}$ with $i>q$ start with positive powers of $v$. As we have already seen, the modified $a_{1}, \cdots, a_{p}$; $b_{1}, \cdots, b_{q}$ will still start with zero powers af $u$.

We now put $v=0$ in (26) and find

$$
1+x y=\left(a_{1} \gamma_{1}+\cdots+a_{p} \gamma_{p}\right)\left(b_{1} \delta_{1}+\cdots+b_{q} \delta_{q}\right) .
$$

As above, it follows that the ratio of any two $a$ 's, or any two $b$ 's, is independent of $u$. Also, $a_{1} b_{1}$ is independent of $u$.

Dividing the first factor in the second member of (26) by $a_{1}$ and multiplying the second factor by $a_{1}$, we find a relation (22) in which $c_{1}=\gamma_{1}=1$. In this relation, $c_{i}$ with $i>p$ and $d_{i}$ with $i>q$ start with positive powers of $u$, whereas, $\gamma_{i}$ with $i>p$ and $\delta_{i}$ with $i>q$ start with positive powers of $v$.

7. Second step. We shall show the existence of a relation

$$
\begin{array}{r}
(1+x y+u v)=\left[1+x y+a_{3}(x, u) \alpha_{3}(y, v)+\cdots+a_{r}(x, u) \alpha_{r}(y, v)\right] \\
\cdot\left[1+b_{2}(x, u) \beta_{2}(y, v)+\cdots+b_{s}(x, u) \beta_{s}(y, v)\right] .
\end{array}
$$

In (30), the $r$ and $s$ may be either the $r$ and $s$ of (21), or the $r$ and $s$ of (21) interchanged. All functions are algebraic, and the four sets

$$
\begin{aligned}
& 1, x, a_{3}, \cdots, a_{r} ; 1, b_{2}, \cdots, b_{s} ; \\
& 1, y, \alpha_{3}, \cdots, \alpha_{r} ; 1, \beta_{2}, \cdots, \beta_{s}
\end{aligned}
$$

are linearly independent. 
We start from the relation (22), which we shall write in the form (21). That is, in (21), we understand that certain terms are free of $u$ and $v$, and that in the remaining terms the $\alpha$ 's and $\beta$ 's have expansions which start with positive powers of $v$. The fact that the remaining $a$ 's and $b$ 's start with positive powers of $u$, we shall not make use of. We shall also, at times, refei directly to the representation (22).

We write $Y=x y, U=u v$, so that (21) becomes

$$
1+Y+U=\left[\sum_{i=1}^{r} a_{i}\left(x, \frac{U}{v}\right) \alpha_{i}\left(\frac{Y}{x}, v\right)\right]\left[\sum_{i=1}^{s} b_{i}\left(x, \frac{U}{v}\right) \beta_{i}\left(\frac{Y}{x}, v\right)\right] .
$$

It is our plan to expand all functions in the second member of (31) in ascending powers of $v$ about $v=0$. For the $\alpha$ 's and $\beta$ 's, we use the same branches that we used above, replacing $y$ by $Y / x$. As regards the $a$ 's and $b$ 's, we use any set of branches associated with each other in (21); that is, we develop such a set of branches about $u=\infty$, and replace $u$ by $U / v$. Each $a$ and $b$ will develop into ascending powers of $v / U$, with coefficients algebraic in $x$.

Suppose then that we have found a development of the second member of (31) in powers of $v$, with coefficients involving $Y, x, U$. The lowest power of $v$ in this development must be $v^{0}$, and the coefficient of $v^{0}$ must be $1+Y+U$. Hence $1+Y+U$ is the product of the coefficients of the lowest powers of $v$ in the two factors of the second member of (31).

If $v^{0}$ is the lowest power in the development of the first factor, then $v^{-}$ is the lowest power in the second factor.

We shall prove that the coefficient of $v^{0}$ in the first factor is not zero. That coefficient is

$$
c_{1}(x) \gamma_{1}\left(\frac{Y}{x}\right)+\cdots+c_{p}(x) \gamma_{p}\left(\frac{Y}{x}\right)+\begin{gathered}
\text { terms involving } \\
\text { positive powers of } U .
\end{gathered}
$$

The $c$ 's and $\gamma$ 's are those which appear in (22). The "terms involving positive powers of $U$ " are those which come from terms in (31) in which the $\alpha$ starts with a positive power of $v$. Such a term must contain a positive power of $U$, because it is a product of a term in the $\alpha$ by one in the $a$. As the $\alpha$ term involves $v$ in a positive power, the $a$ term must involve $v$ in a negative power and hence $U$ in a positive power.

Of course, (32) cannot be zero for every $Y, x, U$. Similarly, the coefficient of $v^{0}$ in the second factor is not zero.

Hence $g \leqq 0,-g \leqq 0$, so that $g=0$. 
Each term in (32) is a non-negative rational power of $U$ multiplied by a product $\rho(x) \sigma(Y / x)$, with $\rho$ and $\sigma$ algebraic. We write (32)

$$
\sum_{i=1}^{h} U^{l_{i}} \rho_{i}(x) \sigma_{i}(Y / x)
$$

where $h$ is some positive integer. The $l$ 's in (33) are not necessarily distinct. We understand that no power of $U$ in (33) has a zero sum for its coefficients. That is, where a sum of coefficients is zero, we suppress the power of $U$. Similarly the coefficient of $v^{0}$ in the second factor in (31) may be written

$$
\sum_{i=1}^{k} U^{m i} \xi_{i}(x) \zeta_{i}(Y / x)
$$

with every $m_{i}$ rational and non-negative and with every $\xi$ and $\zeta$ algebraic. No power of $U$ in (34) has a zero sum for its coefficients.

We have

$$
1+Y+U=\left(\sum_{i=1}^{h} U^{l_{i}} \rho_{i}(x) \sigma_{i}(Y / x)\right)\left(\sum_{i=1}^{k} U^{m_{i} \xi_{i}}(x) \zeta_{i}(Y / x)\right) .
$$

We now develop each $\rho \sigma$ and each $\xi \zeta$ in powers of $x$, for the neighborhood of $x=0$. The development in $x$ of the second member of (35) must start with $(1+Y+U) x^{0}$. Let $x^{o}$ be the lowest power in the first factor, $x^{-0}$ that in the second factor. Multiplying together the coefficients of these lowest powers, we get a relation

$$
1+Y+U=\left(\sum H_{i j} U^{l_{i}} Y^{\lambda_{j}}\right)\left(\sum K_{i j} U^{m_{i}} Y^{\mu_{j}}\right),
$$

with $l$ 's and $m$ 's which are also present in (35), with constant $H$ 's and $K$ 's, and with rational $\lambda$ 's and $\mu$ 's.

Let $t$ be a positive common denominator for the non-negative rational numbers $l$ and $m$ in (36). Let $\tau$ be a positive common denominator for the rational (not necessarily positive) $\lambda$ 's and $\mu$ 's. Let us replace $U$ by $U^{t}$, $Y$ by $Y^{\tau}$ in (36). From (36) it follows that if no $l_{i}$ or $m_{i}$ is unity, $1+Y^{\tau}+U^{t}$, considered as a polynomial in $U$, is reducible in the field of $Y$ and all constants. But because $1+Y^{\tau}$, considered as a function of $Y$, has simple zeros at the $\tau$ th roots of -1 , the equation $1+Y^{\tau}+U^{t}=0$ defines $U$ as a function of $Y$ of $t$ branches, so that $1+Y^{r}+U^{t}$ is irreducible.

We conclude that some $l_{i}$ or some $m_{i}$ in (36) equals unity. Suppose that some $l_{i}$ is unity. As such an $l_{i}$ is also present in (35), we see, since the highest power of $U$ in the first member of (35) is $U$, that no $m_{i}$ is positive. Thus every $m_{i}$ is zero. That is, the second factor of (35) is the

$$
d_{1}(x) \delta_{1}(y)+\cdots+d_{q}(x) \delta_{q}(y)
$$


of (22), with $y$ replaced by $Y / x$. Let the coefficient of $U$ in the first factor of (35) be denoted by $P$. Then

$$
1=P\left[d_{1}(x) \delta_{1}(Y / x)+\cdots+d_{q}(x) \delta_{q}(Y / x)\right] .
$$

From Theorem II, it follows that $q=1$. Turning to (23), we have

$$
1+x y=d_{1}(x) \delta_{1}(y)\left[c_{1}(x) \gamma_{1}(y)+\cdots+c_{p}(x) \gamma_{p}(y)\right] .
$$

We must therefore have $p=2$. Furthermore, multiplying the first factor of (22), and dividing the second factor of (22), by $d_{1} \delta_{1}$, we have $1+x y+u v$ exhibited in the form (30).

8. Third step. We shall prove that every $b$ in (30) is independent of $x$, and every $\beta$ independent of $y$.

Suppose that $b_{2}$, for instance, is not independent of $x$. Let $u_{0}$ be a value of $u$ such that $b_{2}\left(x, u_{0}\right)$ is not a constant, and such that no $b$ or $a$ has a singularity at $\left(x, u_{0}\right)$ for every $x$.

Then $b_{2}\left(x, u_{0}\right)$ must have a pole for some value of $x$. Suppose first that there is a pole for a finite value, $h$, of $x$. Of all functions $b_{i}\left(x, u_{0}\right)$ which have poles at $h$, let

$$
b_{i_{1}}\left(x, u_{0}\right), \cdots, b_{i_{p}}\left(x, u_{0}\right)
$$

be those whose poles at $h$ have a maximum order, their developments starting with terms $c_{i_{m}}(x-h)^{\circ}, g<0$. Because of the linear independence of the $\beta$ 's, the second factor in (30), considered as a function of $x$, will, for $u=u_{0}$ and $y$ and $v$ general, have a pole at $h$ with a first term

$$
\left(c_{i_{1}} \beta_{i_{1}}+\cdots+c_{i_{p}} \beta_{i_{p}}\right)(x-h)^{\sigma} .
$$

Hence the first factor must have a zero when $u=u_{0}, x=h$. Then no $a\left(x, u_{0}\right)$ can have a pole at $h$ and

$$
1+h y+a_{3}\left(h, u_{0}\right) \alpha_{3}(y, v)+\cdots+a_{r}\left(h, u_{0}\right) \alpha_{r}(y, v)=0
$$

for $y$ and $v$ general. But (37) expresses a linear dependence among $1, y, \alpha_{3}, \cdots, \alpha_{r}$. We conclude that $b_{2}\left(x, u_{0}\right)$ cannot have a pole for a finite value of $x$.

Suppose now that $b_{2}\left(x, u_{0}\right)$ has a pole at $\infty$. We see, as above, that, for $y$ and $v$ general, the second factor in (30) has a pole at $\infty$. Since $1+x y+u v$. has only a simple pole at $\infty$ (when $y \neq 0$ ), the development at $\infty$ of the first factor in (30) cannot start with a power of $x$ as high as the first power. This means that some of the $a_{i}\left(x, u_{0}\right)$ have poles at $\infty$ with developments which start with the first power of $x$, and that there is a relation of the type

$$
y+c_{i_{1}} \alpha_{i_{1}}(y, v)+\cdots+c_{i_{q}} \alpha_{i_{q}}(y, v)=0 .
$$


This completes the proof that every $b$ is independent of $x$. Similarly every $\beta$ is independent of $y$.

9. Completion of proof. We shall show that $s=1$ in (30). This means that, in (21), either $s=1$ or $r=1$. Theorem III will be proved.

Let $F(u, v)$ be used to represent the second factor in (30). We shall develop every $a$ in (30) in descending powers of $x$ about $x=\infty$. If the coeffcient of a power $x^{h}$ in the development of $a_{i}$ is $c_{i}(u)$, the coefficient of $x^{h}$ in the development of the second member of (30) is*

$$
\left[c_{1}(u) \alpha_{1}+\cdots+c_{r}(u) \alpha_{r}\right] F(u, v) .
$$

As the $\alpha$ 's are linearly independent, and as the first member of (30) involves only $x$ and $x^{0}$, we conclude that every $a$ is a linear integral function of $x$. Similarly, the $\alpha$ 's are linear integral functions of $y$.

Then (30) becomes

$$
1+x y+u v=[x y A(u, v)+x B(u, v)+y C(u, v)+D(u, v)] F(u, v),
$$

where $A, B, C, D, F$ are sums of products of functions of $u$ by functions of $v$.

Equating coefficients of $x y$, we have

$$
1=A(u, v) F(u, v),
$$

so that, by Theorem II, $F(u, v)$, when written in reduced form, has only one term. As $F(u, v)$ is the second member of (30) we have $s=1$ in (30), and the proof of Theorem III is completed.

* We understand that $a_{1}=1, a_{2}=x, \alpha_{1}=1, \alpha_{2}=y$.

Columbia University,

NEW YoRK, N. Y. 